\title{
EchoGéo
}

15 | 2011

La Belgique, État prospère en décomposition au cœur de l'Europe du nord-ouest

\section{Les destins divergents des régionalismes flamands et francophones : une perspective historique}

\section{Geoffrey Pion et Gilles Van Hamme}

\section{(2) OpenEdition}

\section{Journals}

Édition électronique

URL : https://journals.openedition.org/echogeo/12291

DOI : 10.4000/echogeo.12291

ISSN : 1963-1197

Éditeur

Pôle de recherche pour l'organisation et la diffusion de l'information géographique (CNRS UMR 8586)

Référence électronique

Geoffrey Pion et Gilles Van Hamme, «Les destins divergents des régionalismes flamands et

francophones : une perspective historique », EchoGéo [En ligne], 15 | 2011, mis en ligne le 11 avril 2011, consulté le 03 août 2021. URL : http://journals.openedition.org/echogeo/12291 ; DOI : https://doi.org/ 10.4000/echogeo.12291

Ce document a été généré automatiquement le 3 août 2021

EchoGéo est mis à disposition selon les termes de la licence Creative Commons Attribution - Pas d'Utilisation Commerciale - Pas de Modification 4.0 International (CC BY-NC-ND) 


\title{
Les destins divergents des régionalismes flamands et francophones : une perspective historique
}

\author{
Geoffrey Pion et Gilles Van Hamme
}

\section{Introduction}

1 La récente poussée régionaliste en Flandre pose de façon de plus en plus aiguë la question même de l'existence de la Belgique. Cette percée fulgurante portée par le parti indépendantiste de la NV-A a été précédée au cours de l'histoire de la Belgique par plusieurs vagues régionalistes flamandes (1919-21, 1936, 1965-77). Au contraire, au sud du pays, le régionalisme n'a connu qu'un succès éphémère dans les années 1965-75, avant de disparaître.

2 Dans cet article, nous tentons de comprendre ces différentes vagues régionalistes ainsi que leurs fondements politiques, sociaux et géographiques. En effet, les mouvements régionalistes ont connu des poussées plus ou moins fortes et temporaires en s'appuyant sur des bases électorales très différentes à chaque fois, y compris dans leur dimension géographique. Nous tentons aussi d'expliquer comment ces différentes vagues régionalistes se sont à chaque fois rapidement épuisées. Toutefois, l'irrégularité et l'instabilité des mouvements politiques régionalistes - qui sont comme une série de brutaux accès de fièvre - ne doivent pas masquer la montée structurelle du régionalisme flamand en tant que revendication politique. Cette instabilité ne doit pas non plus masquer les différences de plus en plus profondes qui peuvent exister entre les sociétés du nord et du sud du pays. Cet article a donc pour objectif d'éclairer le paradoxe entre irrégularité des poussées régionalistes et lente dislocation de la Belgique. 
3 Sans rentrer dans le débat autour du concept de partis régionalistes, autonomiste ou ethno-régionaliste (Seiler, 2003 ; De Winter \& Türsan, 1998), nous considérerons dans cet article comme partis régionalistes ceux retenus pour la Belgique par Delwit (2005) dans son chapitre sur l'essor des partis régionalistes en Europe : la Volskunie, le RW (Rassemblement Wallon), le FDF (Front des Francophones), le Vlaams Blok/Belang. Mais nous tiendrons aussi compte d'une série de formations, qui n'ont pas ou peu réussi électoralement, mais qui se rapportent selon nous à l'un ou l'autre des cinq types de partis ethno-régionalistes définis par De Winter \& Türan (1998) : le Frontpartij, le VNV, le RWF (Rassemblement Wallonie France), le Bloc Wallon, Wallonie d'Abord, ProBXL et la Lijst Dedecker (LDD). Ce qui distingue l'ensemble de ces partis n'est pas nécessairement la radicalité de leur revendication régionaliste - qui peut se retrouver dans les partis dits traditionnels, notamment le CD\&V du côté flamand -, mais plutôt de se structurer d'abord autour de la revendication régionaliste, quelle que soit sa nature et son intensité.

4 Après avoir rappelé l'origine des régionalismes et surtout les premières poussées régionalistes en Belgique (section 1), l'article analysera plus en profondeur les fortes poussées régionalistes des années 1965-75 qui ont abouti in fine à la mise en place d'un État fédéral (section 2), avant de s'intéresser aux tendances plus récentes des régionalismes et des formes différentes qu'il prend au nord et au sud du pays (section 3). La conclusion posera la question de l'avenir incertain des régionalismes dans le cadre de l'avenir incertain de la Belgique.

\section{Aux origines des mouvements régionalistes}

5 L'État Belge naît en 1830 de l'alliance improbable de forces sociales et politiques contradictoires (clergé, petite bourgeoisie urbaine, une partie de la grande bourgeoisie) plus que d'une tendance politique de long terme (Vandermotten, 2007). Ce "péché originel » n'a jamais pu être compensé par les politiques visant à renforcer le sentiment national, notamment à travers la construction de grands mythes nationaux. Il ne faut pourtant pas tomber ici dans l'anachronisme: en 1914, lorsque la Belgique s'engage dans la Grande Guerre suite à l'invasion allemande, le sentiment patriotique y est puissant. On peut donc lire l'histoire de la Belgique comme celle d'une faillite, parce que rien n'a permis de transformer un État né de circonstances particulières en véritable État-nation abouti, mais aussi comme un succès remarquable qui se traduit par l'extraordinaire lenteur de la décomposition.

6 L'État belge issu de la révolution de 1830 est un État centralisateur dominé par l'élite francophone qui imposa le français comme langue unique, ce qui a relégué l'utilisation des dialectes flamands et wallons dans la sphère privée. En effet, malgré les libertés linguistiques reconnues explicitement dans la Constitution adoptée en 1831, le flamand ne sera considéré dans les faits que comme un patois et ne sera pas utilisé dans l'Administration (Witte \& Craeybeckx, 1987). Mais il ne faut pas d'emblée lire cet état de fait comme un état d'oppression purement linguistique : les droits du peuple ne sont pas plus pris en considération sur les plans politique, économique ou social qu'ils ne le sont sur le plan linguistique (Gubin, 1982; Miroir \& Leton, 1999). L'évolution de Bruxelles reflète à merveille la nature de l'État belge : en tant que lieu de concentration $\mathrm{du}$ pouvoir politique et économique, Bruxelles - ville historiquement flamande - 
connaît une francisation intense au cours du XIX ${ }^{\mathrm{e}}$ siècle, de moins de $20 \%$ en 1840 à plus de $70 \%$ d'après les évaluations actuelles ${ }^{1}$ (Marissal et al., 2007 ; Lambert, 2010).

7 La crise que traverse la Flandre pendant une bonne partie du XIX ${ }^{e}$ siècle, surtout la Flandre historique (actuelles Flandres Occidentale et Orientale), va progressivement accentuer le fossé qui existe entre des élites largement francisées et une population pauvre pratiquant exclusivement le dialecte flamand (Marissal et al, 2007). Contrairement à ce qu'on observe à Bruxelles, il n'y a pas de francisation de la Flandre et cela s'explique largement par la marginalisation de la région au cours du XIX ${ }^{\mathrm{e}}$ siècle. Pourtant, plus que les masses rurales pauvres, c'est la petite bourgeoisie urbaine flamande qui est la principale victime de la domination culturelle francophone : elle se trouve bloquée dans son ascension au sein de l'administration belge par sa méconnaissance du français, en même temps qu'elle est très largement exclue de la vie politique par le suffrage censitaire. Cette double exclusion politique et culturelle en fera une classe contestatrice (Gubin, 1982). A la fin du XIX siècle, avec l'émergence d'un patronat flamand qui ne doit pas grand-chose à la Belgique nait aussi le souci de faire émerger une élite flamande, qui soit autonome de l'influence francophone (Marissal et al., 2007).

8 Pourtant, malgré quelques succès, le mouvement flamand ${ }^{2}$ est incapable de présenter un front uni face à la classe politique francophone. Sur le plan social, sa base ne s'élargit que lentement au-delà de la petite bourgeoisie urbaine de Flandre. Sur le plan politique, la politisation du mouvement flamand a été assez tardive et ne s'est réellement affirmée qu'après l'échec des stratégies non politiques qui avaient notamment abouti à la « commission des griefs flamands » dont les recommandations ne furent guère appliquées (1857). De plus, les militants du mouvement flamand se sont contentés, en-dehors de quelques épisodes, de former des courants au sein des deux grands partis (libéraux et catholiques) au XIX siècle (Witte \& Craeybeckx, 1986). Le bref épisode du Meeting Partij est une exception, et représente la première expression politique structurée du mouvement flamand dans les années 1860. Il est né à Anvers d'une union des bourgeoisies catholiques et libérales, en opposition avec la construction de fortifications par le gouvernement et par le roi afin de garantir la neutralité absolue de l'Etat belge.... A l'aube du XXe siècle, le Parti daensiste constitue un nouvel épisode important. Ce mouvement ouvrier catholique, issu d'une scission du Parti catholique, est le premier à structurer les revendications flamandes au sein de la classe ouvrière. Surtout implanté dans les régions textiles, le daensisme illustre à merveille à quel point l'oppression sociale et linguistique se confondait en Flandre. Ce parti sera finalement réintégré dans l'aile ouvrière du Parti catholique dès avant la Première Guerre Mondiale. En dehors de ces épisodes, le mouvement flamingant restera longtemps modéré et marginal, au point que la force du sentiment national dont il fait preuve restera un caractère marquant jusqu'en 1914. En effet, ses revendications portent sur l'égalité linguistique et plus précisément le bilinguisme généralisé et s'inscrivent donc pleinement dans le cadre de l'État belge, illustrant à quel point l'État belge était une réalité forte en 1914, même si les fondements de la « dislocation future » ont déjà été mis en place (Gubin, 1982).

9 Malgré des revendications modérées, le mouvement flamand s'est longtemps heurté à un front de refus de la part de la classe politique francophone. Les quelques succès législatifs obtenus avant la Première Guerre Mondiale ne manqueront pourtant pas de susciter une vive réaction. En effet, face à la menace que représente pour la petite 
bourgeoisie wallonne la perte des privilèges francophones en matière d'emploi public, un premier mouvement wallon se structure avec ses manifestations populaires, ses fêtes... Le paradoxe est que, dès 1912, ce mouvement qui ne compte pourtant que quelques centaines de militants cherche à assurer à la Wallonie une autonomie par l'unilinguisme régional (à chaque région sa langue), revendication qui dépasse donc celle du mouvement flamingant à la même époque (Lothe, 1976).

La Première Guerre Mondiale constitue une première rupture, au moins sur le plan symbolique. La surreprésentation des francophones au sein des officiers et des Flamands parmi l'infanterie met en exergue la violence de la domination francophone au sein de l'État belge. Il en résultera le premier parti nationaliste flamand, le parti frontiste (Frontpartij) qui glane cinq députés lors des élections fédérales de 1919 et connaît un succès électoral important dans la décennie suivante (Van Haute, 2005). Pourtant dans l'euphorie de la victoire et l'union nationale qui s'en suit, les frontistes ne parviennent pas à étendre leur base électorale et à s'imposer au-delà de la zone du front de l'Yser ${ }^{3}$, où leurs scores dépassaient $10 \%$ du corps électoral (Marissal et al., 2007).

$11 \mathrm{Au}$ cours des années 1930, il faut souligner que l'égalité culturelle est finalement acquise pour les Flamands, notamment avec la flamandisation complète de l'Université de Gand en 1931 et avec l'adoption en 1932 du principe de l'unilinguisme en Flandre et en Wallonie et du bilinguisme à Bruxelles. Mais, tout se passe comme s'il était déjà trop tard, le mouvement flamingant semble déjà trop ancré dans la société flamande.

Les années 1930 marquent un nouveau tournant avec les succès de l'extrême-droite néerlandophone régionaliste au nord du pays (VNV). Le parti trouve son soutien électoral dans les zones périphériques et rurales à forte dominante catholique de Flandre historique d'une part, de Campine et du Limbourg d'autre part (illustration 1). En revanche, dans les grandes villes flamandes, le VNV trouve peu d'écho, y compris à Anvers qui fut et sera pourtant le grand bastion du régionalisme flamand. A la même période, le mouvement d'extrême droite REX réalise d'encore meilleurs scores au sud du pays ainsi qu'à Bruxelles mais défend à l'inverse du VNV un unitarisme intransigeant. 


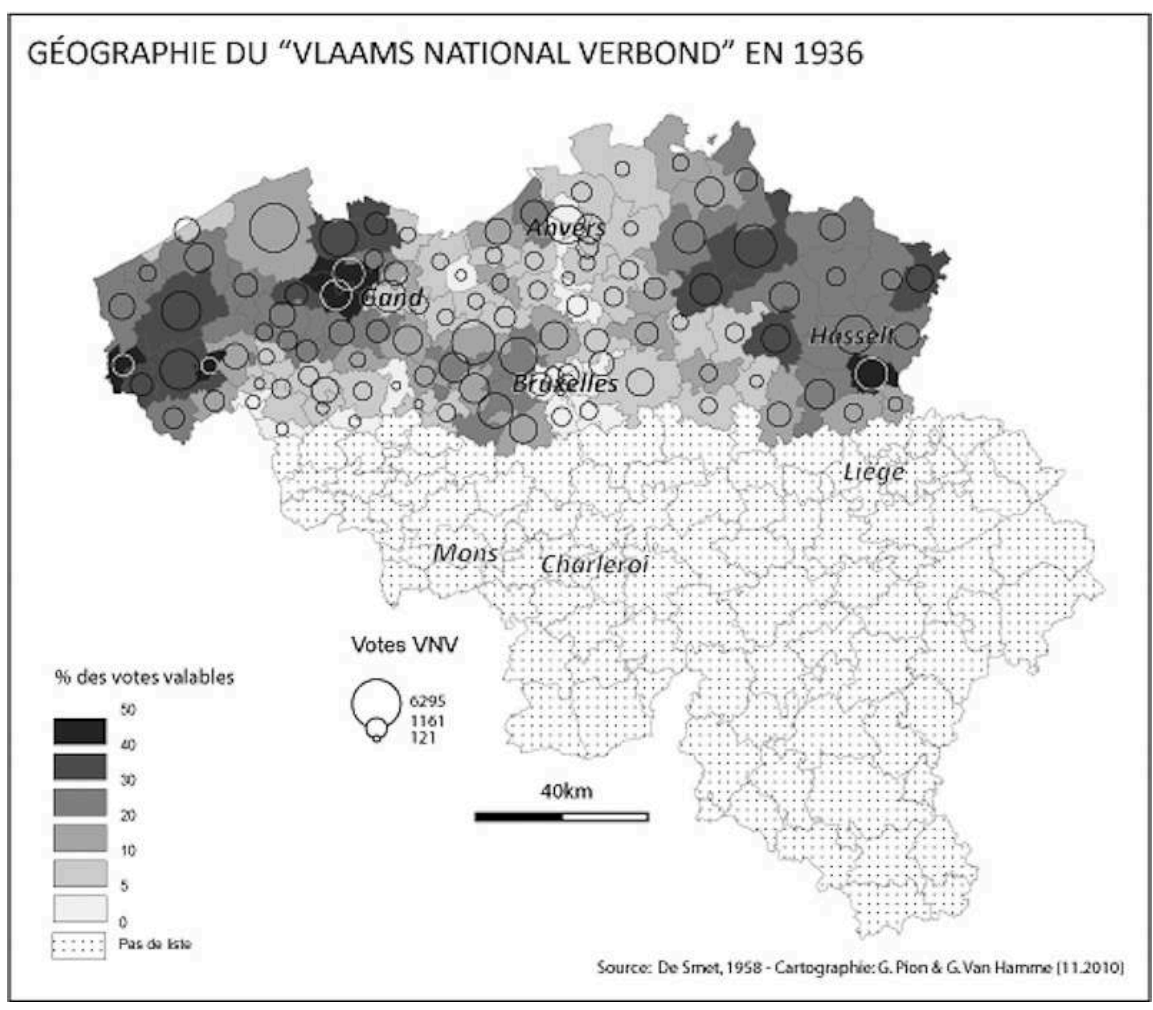

Source : De Smet, 1958 - Cartographie : G. Pion et G. Van Hamme, 2010.

13 Il faut souligner que le VNV est difficilement assimilable à un parti fasciste, sa composante autonomiste radicale étant sa principale caractéristique. Sa collaboration avec l'occupant allemand relève plus de la volonté de faire émerger la nation flamande que d'une adhésion idéologique, même si les segments les plus radicaux seront bien entendu soutenus par l'occupant et que le VNV évoluera vers des positions nationalessocialistes durant la guerre (Gilissen, 1951). La collaboration du mouvement nationaliste flamand reste toujours un point de litige faisant l'objet de débats et de propositions de lois, et a contribué depuis la fin de la guerre à le discréditer. La revendication d'amnistie constitue ainsi toujours un ciment fort non seulement pour l'extrême-droite mais aussi pour une grande partie de la classe politique et du peuple flamand (Gijsels \& Vander Velpen, 1992).

\section{Les grandes vagues régionalistes des années 1960 et 1970}

Malgré le discrédit du mouvement flamingant, la période d'immédiat après-guerre se traduit par une succession de crises majeures qui révèlent l'intensité du clivage communautaire. Dès 1950, la question royale porte sur le retour du Roi Léopold III alors que lui est reprochée son attitude bienveillante vis-à-vis de l'occupant. Elle aboutit à un referendum qui donne la majorité pour un retour du Roi $(57,7 \%)$, mais avec une très forte différence entre le nord et le sud du pays. En Flandre, on se prononce très largement pour ce retour $(72 \%)$ - conformément à une volonté d'amnistie - alors que la Wallonie (ainsi que Bruxelles) s'y oppose ( $42 \%$ ). Pour la première fois, la loi du 
nombre s'impose aux francophones. Pourtant, face au climat insurrectionnel provoqué par la perspective du retour de Léopold III, le roi finit par abdiquer, frustrant la majorité flamande. Ensuite, la guerre scolaire (1950-58) réanimera le clivage clérical/ laïc sur la question des subsides accordés à l'enseignement libre (c'est à dire catholique) très majoritaire en Flandre, et alimente encore le conflit communautaire. Enfin, les grandes grèves de 1960 qui se dressent contre une politique d'austérité seront décisives dans la renaissance et la popularisation du mouvement wallon, dans la mesure où la Wallonie industrielle est au cœur de ce mouvement social. Sur fond de déclin économique, ces grèves illustrent la fusion momentanée de revendications sociales radicales de nature anti-capitaliste et du mouvement wallon.

Ces crises ne portent pas sur un enjeu communautaire mais révèlent l'intensité des différences sociales, politiques et idéologiques entre le nord et le sud du pays: la Flandre est catholique, conservatrice ; la Wallonie est laïque, socialiste et marquée par une forte tradition de lutte ouvrière. En outre, il devient de plus en plus évident que la prospérité wallonne s'essouffle (fermetures des charbonnages dès les années 1950) alors que la Flandre connaît, y compris dans ses zones périphériques, un développement fulgurant. Cette inversion du clivage économique traditionnel s'intensifiera avec la crise des années 1970 et jouera alors un rôle capital.

C'est dans ce contexte qu'(ré)émergent dans les années 1960 des forces politiques régionalistes au nord mais aussi, pour la première fois, à Bruxelles et au sud du pays.

En 1954, des dissidents du parti catholique forment la Volksunie (VU) en Flandre avec pour objectif d'amener dans le champ politique les revendications autonomistes flamandes, y compris des nostalgiques du flamingantisme et d'anciens collaborateurs. Très vite, le parti unit des courants idéologiques très variés se rassemblant autour de la question du fédéralisme et de l'autonomie flamande. Les premiers succès électoraux du parti s'opèrent en 1961. La VU atteint son meilleur score au début des années 1970 et participe au gouvernement entre 1977 et 1979. A l'image du Rassemblement Wallon (RW), la VU opère un tournant à gauche dans les années 1970, et se voit lâchée par sa frange la plus autonomiste et la plus marquée à droite du mouvement (Govaert, 1993). Ces dissidents seront à la fin des années 1970 à la base de la formation du parti d'extrême droite régionaliste flamand, le Vlaams Blok (VB).

17 La Volskunie possède une base électorale relativement interclassiste et sa géographie ne présente pas de zones de force très marquées, même si ses meilleures scores se situent en Flandre occidentale où le VNV des années 1930 était déjà puissant (illustration 2). Mais contrairement à ce parti, les scores de la VU sont relativement faibles dans le Limbourg. Plus fondamentalement, la VU ne présente pas le caractère périphérique du nationalisme flamand d'avant-guerre et réalise de bons scores dans l'axe métropolitain Malines-Anvers, particulièrement dans les cantons autour du grand port flamand, ainsi que dans l'arrondissement de Hal (périphérie bruxelloise située en territoire flamand) où les enjeux communautaires sont exacerbés par la périurbanisation francophone vers la banlieue flamande (Vandermotten, 2007).

En 1968, le Rassemblement Wallon (RW) est créé par des dissidents wallons du Parti Socialiste Belge (PSB) en réaction à l'unitarisme des partis traditionnels. Le parti est clairement fédéraliste et défend un régionalisme culturel et politique (Pilet, 2005). Il s'associe la même année avec le Front Démocratique des Francophones (FDF) qui depuis 1964 défend les intérêts des francophones à Bruxelles et dans sa périphérie. Entre 1968 et 1974 , cette formation glane une part croissante des suffrages pour atteindre près de 
$20 \%$ des votes valables en Wallonie et $35 \%$ à Bruxelles aux élections fédérales 1974 . Suite à ces élections, le RW participe au gouvernement Tindemans II afin de faire progresser encore la régionalisation et met notamment en place le premier conseil régional wallon fin 1974.

Le RW récolte $20 \%$ des votes valables dans la majorité des cantons de la province de Namur ainsi que dans les cantons urbains carolorégiens et liégeois où il dépasse localement $30 \%$ des suffrages, soit globalement dans les zones urbaines où le PSB était bien implanté mais pas complètement hégémonique ou dans l'Entre Sambre et Meuse très francophile (illustration 2). Ses zones de faiblesse correspondent aux territoires ruraux ardennais, hesbignons et du Pays des Collines. Le FDF, lui, se présente dans la province du Brabant et frôle la barre de $50 \%$ des votes valables dans les cantons bourgeois du sud-est de Bruxelles; il est aussi fortement implanté dans les minorités francophones de la banlieue flamande. A la différence des régionalistes flamands et wallons dont les bases sociales sont interclassistes, il réunit un électorat socialement favorisé soucieux de préserver le statut du français notamment dans des territoires peuplés majoritairement de francophones en territoire flamand.

Illustration 2 - Géographie des régionalismes en Belgique en 1974

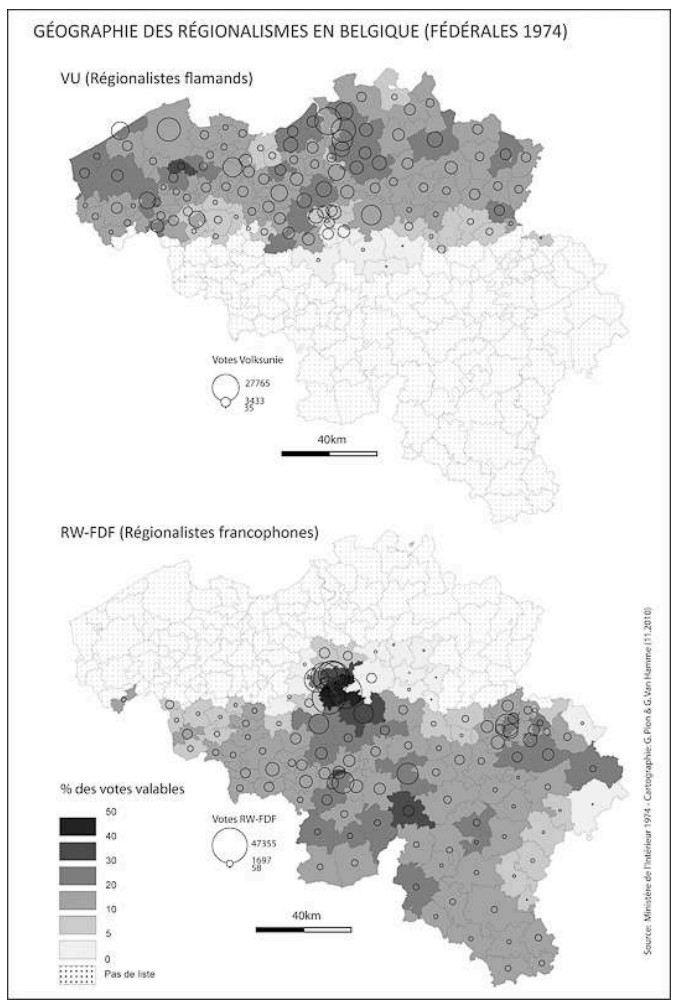

Source : Ministère de l'Intérieur, 1974 - Cartographie : G. Pion et G. Van Hamme, 2010.

Durant près de 20 ans, la Belgique est donc marquée par trois mouvements régionalistes, l'un flamand, les deux autres francophones. Cette poussée régionaliste, autonomiste ou fédéraliste selon le point de vue ou la période, se traduit par une première réforme de l'État en 1970 qui voit la création de trois régions ainsi que trois communautés linguistiques ${ }^{4}$. Elle sera suivie d'une deuxième en 1980 qui crée les Régions wallonnes et flamandes ainsi que les communautés française, flamande et germanophone puis d'une troisième en 1988-89 qui définit le statut de la Région de 
Bruxelles-Capitale. En 1993, la Belgique devient un État fédéral à part entière qui se compose de communautés et de régions (Vandermotten, 2007). Victimes du succès de leurs revendications, ces partis régionalistes vont agoniser plus ou moins rapidement, en particulier le RW qui disparaît du paysage politique dès le milieu des années 1980. Le déclin de ces partis est aussi dû à une évolution politique essentielle, à savoir la scission des grands partis traditionnels - socialiste, catholique et libéraux - sur une base linguistique dans les années $1970^{5}$. Depuis cette date, les partis «frères" s'adressent exclusivement à leur propre communauté, et reprennent à leur compte le discours communautariste des partis régionalistes de part et d'autre de la frontière linguistique.

21 Certes, en Flandre, le régionalisme électoral ne faiblit apparemment pas après 1980, du fait des succès électoraux du VB, parti d'extrême-droite issu d'une dissidence de la VU. Pourtant, bien que le VB ait un discours flamingant radical, c'est-à-dire indépendantiste, il ne fonde pas l'essentiel de ses succès électoraux sur cet enjeu (Billiet, 1998). Comme on l'a déjà suggéré, les réseaux flamingants ont pourtant joué un rôle essentiel, en donnant une structure et une organisation à l'extrême-droite flamande. De plus, celle-ci, à travers les réseaux (et les enjeux) nationalistes, n'a jamais été complètement isolée du reste du monde politique flamand, même si la stratégie du cordon sanitaire (appliquée sur le plan politique mais certes pas dans les médias, auxquels le VB a un accès réel, au contraire de l'extrême-droite francophone) a écarté ce parti de tous les niveaux de pouvoir en Flandre. A l'image du FN en France, le VB bâtira en fait l'essentiel de son succès sur un discours anti-immigré associé à la délinquance et à la dégradation des villes qui trouvera un écho de plus en plus large auprès de la classe ouvrière flamande (De Decker \& al, 2005).

Dans sa géographie aussi, le Vlaams Blok (VB) présente d'évidentes analogies avec le FN français : il connaît ses meilleurs scores auprès de l'électorat populaire dans les zones centrales et urbaines, en particulier dans son bastion d'origine d'Anvers. Il a certes diffusé de façon remarquable à travers le territoire flamand (illustration 3), mais les scores du VB dans les zones périphériques de Flandre sont restés inférieurs à ceux atteints dans ses fiefs initiaux. L'explication de cette géographie centrale se trouve dans le poids du pilier catholique dans les zones périphériques flamandes, où un encadrement social serré a sans doute constitué un obstacle à la pénétration de l'extrême-droite (Van Hamme 2008). De ce point de vue, il est intéressant de constater que la géographie de l'extrême-droite flamande à partir des années 1980 est à l'opposé de celle des années 1930. En effet, à l'époque, dans les années 1930, l'extrême-droite s'appuyait au contraire sur l'ultra-droite catholique. 
Illustration 3 - Évolution de la géographie du vote Vlaams Blok en Flandre

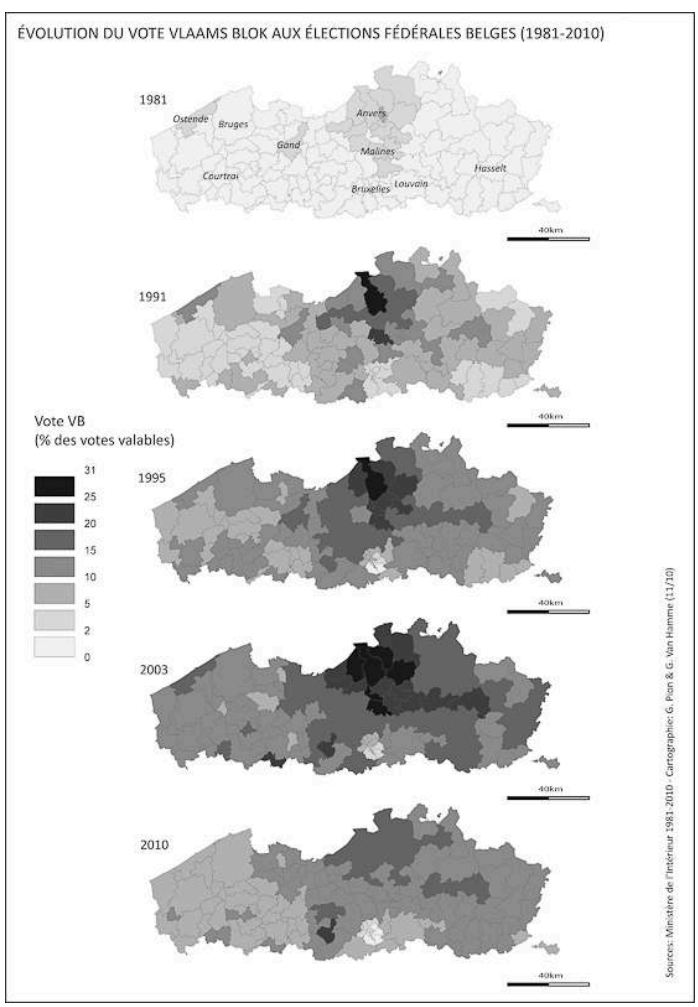

Source : Ministère de l'Intérieur, 1981-2010 - Cartographie : G. Pion et G. Van Hamme, 2010.

La situation est bien différente dans le sud du pays où une extrême-droite fragmentée et politiquement inconsistante défend plus fréquemment l'unitarisme. Quelques dissidences ont bien tenté de jouer sur la fibre régionaliste de l'électeur wallon (AGIR, Bloc Wallon, Wallonie d'Abord), mais sans succès.

\section{La résurrection du régionalisme en Flandre}

Le déclin du régionalisme flamand s'était traduit notamment par la fin de la VU, et la naissance de deux partis régionalistes au début des années 2000 : Spirit représente l'aile gauche alors que la NV-A est issue de l'aile droite du mouvement flamingant. Les débuts sont médiocres et ne leur permettent pas de s'installer véritablement dans le paysage politique flamand. Toutefois, pour la NV-A, la formation d'un cartel avec le parti social chrétien du CD\&V en 2003 va changer la donne ${ }^{6}$. En effet, les succès électoraux croissants du Vlaams Blok depuis les années 1990 et la victoire électorale du cartel CD\&V-NV-A, après une campagne axée sur les enjeux communautaires en Flandre en 2007, ont relancé le débat communautaire. Sous la pression de la fraction régionaliste du cartel social-chrétien, du Vlaams Belang et de la Lijst Dedecker ${ }^{7}$, le monde politique flamand exige alors une nouvelle réforme de l'État belge qui consiste à octroyer toujours plus de compétences aux Régions et aux Communautés et à scinder l'arrondissement électoral de Bruxelles-Hal-Vilvorde (Vandermotten, 2007). A l'issue des élections fédérales de 2007, les discussions qui débouchent sur le gouvernement Leterme I durent plus de six mois et cristallisent les débats politiques nationaux sur la question communautaire. En septembre 2008, le cartel CD\&V-NV-A est rompu et la NVA décide de reprendre seule son destin en main. Lors des élections régionales 2009, la 
NV-A obtient $13 \%$ des suffrages en Flandre et récupère une partie des électeurs Vlaams Belang qui voit son score électoral diminuer pour la première fois depuis ses débuts!

A la suite de la chute du gouvernement ${ }^{8}$, les élections fédérales anticipées de juin 2010 vont quant à elles provoquer un véritable séisme politique en Belgique : la NV-A obtient près de $30 \%$ des votes valables en territoire néerlandophone. Jamais un parti régionaliste flamand n'avait réalisé un tel score et encore moins n'était devenu le premier parti flamand. En plus de la NV-A, à l'occasion de ces élections, deux autres formations clairement régionalistes voire confédéralistes ou purement et simplement indépendantistes se présentaient en Flandre: le Vlaams Belang et la Lijst Dedecker (LDD). Si ces deux partis ont souffert du raz-de-marée en faveur de la NV-A et de son leader Bart De Wever, ils représentent néanmoins encore $13 \%$ de l'électorat! Une fois encore, à la stabilité électorale et politique du côté francophone s'opposent le bouillonnement et l'instabilité du côté flamand.

L'illustration 4 reprend les géographies des trois composantes du régionalisme flamand aux élections fédérales 2010. La LDD, la moins importante, a une géographie en cercles concentriques autour de la ville côtière de Nieuport où se présentait le président et leader emblématique du mouvement, Jean-Marie Dedecker. Contrairement aux autres branches du mouvement flamand, la LDD ne s'appuie pas sur une structure forte et la seule personnalité de son leader ne suffit pas à créer un parti dont l'implantation territoriale dépasse la zone d'influence de son leader. Toutefois, il est intéressant de noter que ce parti s'implante dans une zone de faiblesse du mouvement flamand actuel. Le Vlaams Belang quant à lui connaît à cette occasion sa première grande défaite électorale même s'il garde un score élevé dans un paysage politique flamand très éclaté. Comme souvent lors d'une défaite électorale, le parti résiste mieux dans ses fiefs, autour d'Anvers ainsi que dans les régions d'Alost et de Beringen (illustration 4). Enfin la NV-A réalise une percée généralisée sur le territoire flamand mais les progrès les plus importants se situent dans le nord-est du pays (Campine, Limbourg), justement dans des zones où le Vlaams Belang avait connu des percées récentes mais manifestement fragiles. 
Illustration 4 - Géographie des partis régionalistes en 2010

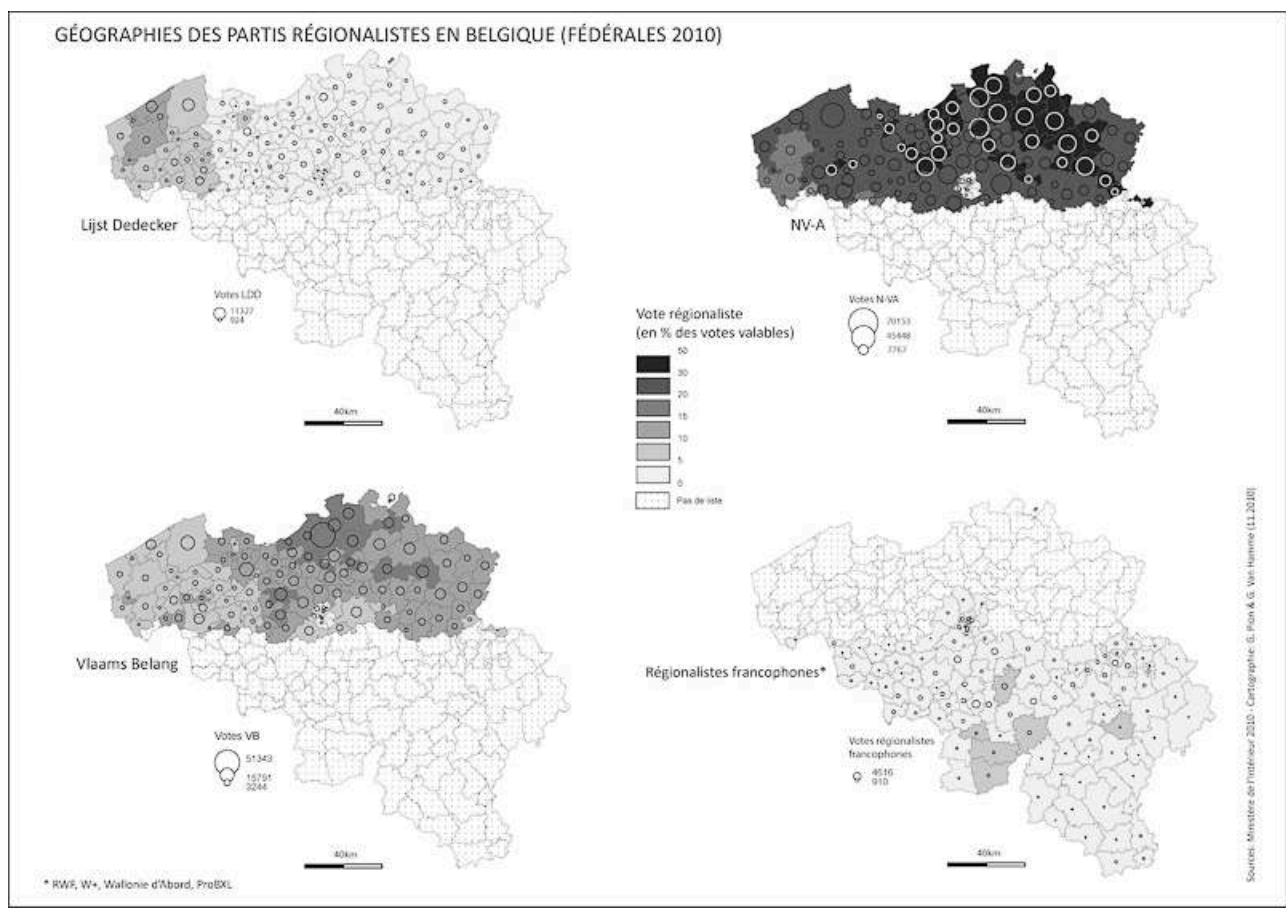

Source : Ministère de l'Intérieur, 2010 - Cartographie : G. Pion et G. Van Hamme, 2010.

Comment réagissent la classe politique et la société civile francophone à ces revendications flamandes? Contrairement aux années 1970, où le fédéralisme et le régionalisme étaient défendus par une partie du spectre électoral francophone, la défense des intérêts francophones est désormais assurée par les quatre grands partis francophones: PS (socialiste), MR (libéral), cdH (social-chrétien) et Ecolo. Si le MR associé en cartel au Front des Francophones à Bruxelles est le parti qui marque la plus franche opposition aux revendications flamandes, il peut être étonnant de constater qu'aucune surenchère régionaliste n'a lieu en Wallonie et à Bruxelles, ou presque. En effet, lors des élections fédérales 2010, trois formations régionalistes ou rattachistes se présentaient en Wallonie : le Rassemblement Wallonie France, Wallonie + et le parti Wallonie d'Abord, ce dernier formé par d'anciens membres de l'extrême-droite francophone. Même additionnés, leurs résultats électoraux sont faibles et ne dépassent les $5 \%$ des votes valables que dans quelques cantons de la province de Namur (illustration 4). A Bruxelles, la thématique de défense des intérêts francophones de la région capitale et de la périphérie est toujours essentiellement défendue par le FDF mais en association avec le PRL-MR9. Nous n'avons donc plus de résultats spécifiques pour cette composante du mouvement libéral francophone depuis 1991, ce qui constitue évidemment un biais dans les illustrations 4 et 5 .

Mais la différence avec les poussées régionalistes des années 1960-70 est plus profonde encore tant la population francophone du pays est restée unitariste et donc très peu réceptive aux revendications autonomistes flamandes. C'est bien ce contraste dans la conception même que les deux communautés ont sur ce que doit devenir l'État belge qui rend très difficile une sortie de crise : sur une échelle de 0 à 10 où 0 représenterait le transfert total des compétences aux Régions, 5 le statu quo et 10 toutes les compétences à l'État central, les Flamands ont une moyenne de 4,28 et les francophones de 5,25 (Partirep $\left.{ }^{10}, 2009\right)$. En Flandre, l'idée d'un confédéralisme dans lequel l'État 
central ne garderait que quelques compétences symboliques semble donc sinon dominante ${ }^{11}$, au moins bien implantée. Du côté francophone, la volonté de maintenir des liens forts entre le nord et le sud du pays, notamment en matière de solidarité interpersonnelle, parait ultra-majoritaire. Dans ce contexte, même les partis régionalistes wallons traduisent plutôt la recherche anticipative d'alternatives à la Belgique qu'une volonté indépendantiste. C'est ainsi que le Rassemblement Wallonie France, au lieu de tenter de retarder l'échéance de la réforme de l'État (ou d'en limiter la portée) comme le font les partis traditionnels francophones, propose de «faire de la Wallonie, et de Bruxelles si elle le désire, une région de France à part entière ».

Pourquoi ce contraste? Nous proposons ici trois pistes explicatives complémentaires pour tenter de comprendre les sorts divergents des régionalismes au nord et au sud du pays.

Premièrement, cette différence met en lumière un net contraste en termes d'identité. Il semble qu'existe une nation flamande, au moins une identification à un ensemble régional bien délimité alors que rien de tel ne semble exister du côté wallon (ou francophone ?). C'est ainsi que du côté flamand, $44 \%$ des personnes s'identifient d'abord à la Belgique contre $55 \%$ du côté wallon ${ }^{12}$. L'identification première à la Flandre concerne $26 \%$ des personnes interrogées, soit un score tout de même nettement inférieur à celui du pays ${ }^{13}$. Du côté wallon, l'identification régionale est à la fois plus floue et plus faible : $10 \%$ des personnes s'identifient prioritairement à la Wallonie alors que $4 \%$ privilégient l'ensemble francophone et que l'identité européenne y semble plus forte qu'en Flandre (16 contre $9 \%$ ). Comment expliquer ces différences ? Comme on l'a déjà souligné, la Flandre - en tout cas une grande partie de la société flamande - ne doit pas grand-chose à la Belgique : c'est le développement autonome dès la fin du XIX ${ }^{e}$ siècle et surtout après la Seconde Guerre Mondiale d'un petit patronat en Flandre historique qui a permis à la région de lentement récupérer de la crise du textile; de plus, Anvers, bien qu'ayant bénéficié de la prospérité de la Belgique, de son aventure coloniale et des investissements publics dans son port, est naturellement tournée vers l'international et son élite est en fin de compte assez peu (ou moins que les autres) intégrée à la nation belge ${ }^{14}$; enfin, après-guerre, le développement fulgurant de la Flandre repose aussi en grande partie sur des investissements étrangers, en particulier dans le Nord-est mais aussi dans les grandes villes. En revanche, la Wallonie voit d'emblée son destin lié à celui de la Belgique et, plus précisément de la grande finance bruxelloise. Non seulement, la petite bourgeoisie wallonne a pu s'intégrer au sein de l'Administration. Mais surtout, les développements miniers et industriels précoces du Hainaut et de Liège ont très rapidement été contrôlés par les grands groupes financiers implantés à Bruxelles (dès les années 1840), si bien que la grande bourgeoisie wallonne a été réduite à la portion congrue. L'éphémère puissance du mouvement wallon dans les années 1960 a correspondu à une période où s'amoncelaient les menaces sur la prospérité wallonne, en particulier celle de sa grande industrie. Le déclin économique violent et irréversible à partir des années 1970 semble avoir sonné le glas d'un régionalisme «offensif» et renforcé progressivement la conviction que l'État belge était le meilleur garant pour l'avenir en Wallonie. Plus précisément, si on a pu penser un temps que l'identité wallonne pourrait se construire à partir du mouvement ouvrier et des luttes ouvrières - en particulier au cours des grandes grèves de 1960 - le déclin de la classe ouvrière wallonne a mis fin à ce processus (Hasquin, 1976). 
La deuxième explication relève d'une combinaison de l'analyse de l'offre électorale et de l'évolution des comportements électoraux depuis les années 1970. Nous faisons ainsi l'hypothèse que du côté flamand, les classes les plus menacées par les bouleversements économiques avaient pu trouver une réponse à leurs difficultés dans les partis structurés à partir du mouvement flamand: c'est en particulier le cas du Vlaams Belang, caractérisé par un électorat ouvrier et peu diplômé de la Flandre urbaine et métropolitaine en voie de désindustrialisation (Van Hamme, 2008; De Decker \& al, 2005). Socialement, les électeurs du NV-A ne répondent pas à ce profil, puisque le parti trouve au contraire ses appuis au sein des employés et des personnes dont le diplôme est élevé. Il semble que ce parti présente une offre acceptable et structurée à des classes supérieures radicalisées pour lesquelles un vote en faveur du Vlaams Blok/Belang ${ }^{15}$ est impensable. Du côté wallon, en revanche, en l'absence d'offre alternative crédible aux partis traditionnels, et dans un contexte peu porteur pour un mouvement wallon vindicatif, ces classes menacées et/ou radicalisées se sont réfugiées dans l'abstention ${ }^{16}$, le vote blanc et nul ou de petits partis sans espoir de représentation au dessus de l'échelon local (y compris en 2010 le FN qui depuis 2009 n'a plus aucun député régional, communautaire ou fédéral). Ces évolutions sont illustrées sur la figure $5:$ du côté francophone, les partis régionalistes réalisent des scores inférieurs à $5 \%$ des inscrits depuis 20 ans mais les votes « marginaux ${ }^{17}$, y compris l'abstention et les votes blancs et nuls, ont graduellement augmenté pour atteindre plus de $25 \%$ des inscrits; du côté flamand, ces derniers représentent une proportion constante et modérée des votants alors que les partis régionalistes ont des scores de plus en plus élevés. On notera aussi l'importance prise par le VB parmi les votes régionalistes depuis le début des années 1990 , tout en rappelant que leur succès électoral repose d'abord sur d'autres enjeux que le régionalisme.

Illustration 5 - Évolutions des votes marginaux et régionalistes en Belgique depuis les années 1930

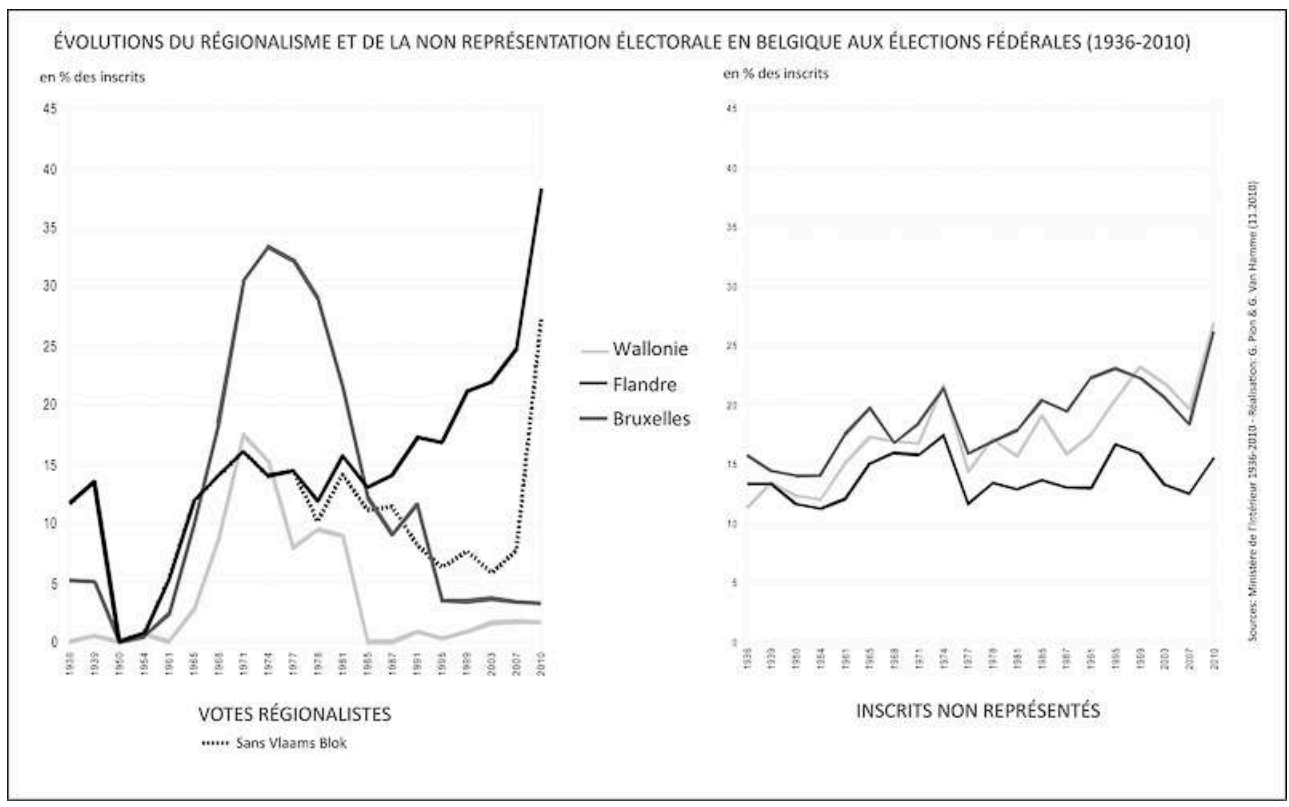

Source : Ministère de l'Intérieur, 1936-2010 - Réalisation : G. Pion et G. Van Hamme, 2010

L'illustration 6 confirme ce constat: la carte des régionalismes y est quasiment le négatif de celui de la marginalité électorale en 2010, au point que la frontière 
linguistique est presque parfaitement dessinée sur ces cartes. Il semble donc en analysant l'histoire électorale belge depuis les années 1930 qu'à la manière de nombreux autres pays occidentaux, la communauté française se détourne de la politique, alors que la Flandre, elle, n'a pas connu de baisse de la participation électorale mais accorde des scores de plus en plus élevés aux partis régionalistes radicaux et/ou d'extrême-droite.

Enfin, la troisième explication peut se résumer simplement à la notion d'égoïsme économique régional, que l'on retrouve dans d'autres régionalismes européens notamment en Italie du Nord. Après des décennies de crise en Wallonie et de croissance en Flandre, l'écart économique est important entre les deux régions. Il en résulte des transferts significatifs du nord au sud du pays, même s'il n'existe pas de véritable accord sur leur montant et que le rôle économique joué par la Région de BruxellesCapitale - vers laquelle affluent chaque matin plus de 300000 navetteurs (dont deux tiers venus de Flandre) - contribue encore à opacifier la situation. Ces transferts sont au cœur des revendications flamandes pour l'autonomie fiscale, voire la régionalisation de la sécurité sociale.

Illustration 6 - Géographies du régionalisme et des votes marginaux en 2010

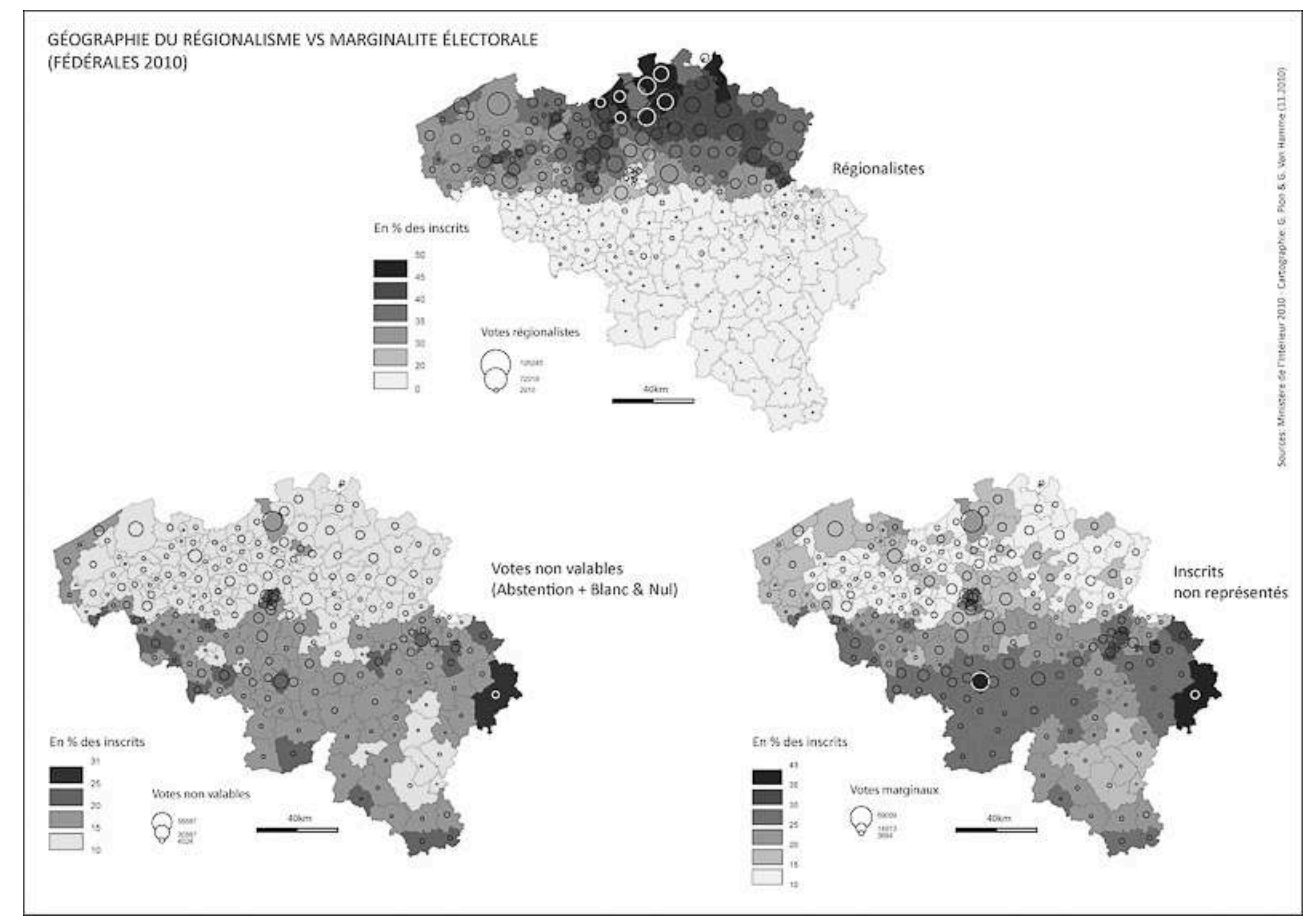

Source : Ministère de l'Intérieur, 2010 - Cartographie : G. Pion et G. Van Hamme, 2010.

\section{Conclusion}

Cette remontée dans le temps permet de mieux comprendre l'ampleur du phénomène régionaliste en Flandre. Depuis les revendications linguistiques de la petite bourgeoisie flamande au XIX ${ }^{\mathrm{e}}$ siècle, le flamingantisme a connu des évolutions importantes qui se sont traduites par plusieurs poussées électorales depuis la Première Guerre Mondiale (1921, 1936, années 1965-75, 2009- ?). Les années de l'entre-deux-guerres avaient vu l'inscription forte à l'agenda politique des revendications linguistiques et culturelles 
flamandes. Une fois les droits culturels et linguistiques obtenus et passées les séquelles de la guerre sur le mouvement flamand, les années 1960 ont vu la montée de revendications fédéralistes et régionalistes. Les vagues régionalistes du sud et du nord du pays des années 1965-75 ont alors abouti aux trois premières réformes de l'Etat belge entre 1970 et 1985 et à la mise en place d'un État fédéral. En 1993, une quatrième réforme de l'Etat fixe le paysage institutionnel de la Belgique jusqu'à la crise récente ${ }^{18}$. En fin de compte, le succès même des revendications régionalistes et leur prise en compte par les partis traditionnels après leur scission sur une base linguistique aboutissent au déclin des partis régionalistes.

Pourquoi alors une nouvelle crise à partir de 2007? Nous avons proposé trois explications complémentaires à cette question. D’abord, les poussées régionalistes flamandes représentent des accès de fièvre certes brefs mais reposent en fait sur une montée structurelle de l'idée même de régionalisme flamand. Tout se passe comme si la lente progression des revendications flamandes ne trouvant pas de réponse politique suffisante aboutirait à de soudaines poussées de partis régionalistes qui, une fois leur mission accomplie, sombreraient dans le déclin. C'est ainsi qu'après l'équilibre temporaire trouvé au début des années 1990, le régionalisme politique radical n'est plus porté que par le parti d'extrême droite Vlaams Blok/Belang alors même que celui-ci n'a pas bâti son succès sur cette thématique mais plutôt sur les enjeux classiques de l'extrême-droite, essentiellement l'immigration. Au début des années 2000, les tensions réapparaissent et se cristallisent en particulier autour de la question de la scission de l'arrondissement électoral encore bilingue de Bruxelles-Hal-Vilvorde ${ }^{19}$. Mais la revendication est plus profonde et l'idée d'une nouvelle grande réforme de l'Etat monte en Flandre. Face au blocage politique - et une première période de 6 mois sans gouvernement après les élections de 2007 -, à l'incapacité des partis traditionnels flamands à imposer leur vue et à la surdité des partis francophones aux volontés flamandes, les partis régionalistes flamands ont vu leur score monter en flèche aux élections régionales de 2009 et fédérales de 2010 , où ils recueillent près de $45 \%$ des votes valables dont $30 \%$ au seul NV-A. Ensuite, nous avons développé l'hypothèse que les partis régionalistes et/ou d'extrême-droite en Flandre sont parvenus à capter un électorat socialement menacé et/ou contestataire, ce qui explique le niveau relativement bas de votes "marginaux ». On a aussi montré que si une telle offre a pu se développer de façon structurée en Flandre, en particulier celle de l'extrême-droite, c'est sur base des réseaux nationalistes flamands historiques. En revanche, du côté francophone, en l'absence d'offre crédible pour cet électorat, on observe une poussée tendancielle de votes "marginaux ». Enfin, les écarts économiques croissants entre le nord et le sud du pays ont nourri le régionalisme flamand, qui insiste en permanence sur le coût de la Wallonie pour les Flamands.

Alors que les négociations en vue de former un gouvernement sont au point mort depuis plusieurs mois, on peut se demander plus que jamais ce que sera l'avenir de la Belgique ? Si les partis francophones ont tenté dans un premier temps de résister à une nouvelle grande réforme de l'Etat, se rendant par là en partie responsable de la radicalisation en Flandre, la situation est désormais différente. Alors que les partis francophones ont fini par accepter l'idée d'une grande réforme de l'Etat, le parti régionaliste de la NV-A doit faire face à des choix d'autant plus difficiles qu'il ne semblait pas vraiment préparé à se trouver en position de force. En effet, tout compromis même favorable mettrait le parti dans une position délicate pour deux 
raisons. D'une part, un tel compromis serait de toute façon inacceptable pour les militants et cadres radicaux au cœur du parti. C'est exactement un compromis de ce type qui avait mené à la fin des années 1970 une partie de la Volksunie à faire dissidence pour fonder le Vlaams Blok. D'autre part, comme on l'a observé par le passé, un succès politique sur ses revendications menacerait la raison d'être de ce parti et pourrait affaiblir considérablement sa base électorale. Dès lors, seule une montée de l'indépendantisme pur et simple - objectif à terme des partis régionalistes flamands comme la NV-A - pourrait lui permettre de maintenir son poids politique.

On peut penser que la nouvelle réforme de l'Etat et le transfert de nouvelles compétences aux Régions et Communautés va se réaliser dans les mois ou les années qui viennent. Mais alors, l'espace se réduira entre la configuration institutionnelle de l'État belge et l'éclatement du pays. Autrement dit, si cette réforme aboutit, toute future nouvelle grande réforme - qui sera probablement précédée d'une nouvelle grande vague régionaliste qui déferlera peut-être aussi en Wallonie - devra s'attaquer aux derniers fondements de l'Etat central: les fonctions régaliennes et la solidarité interpersonnelle. Deux options s'offriront alors à la Belgique : celle d'une coquille vidée de tout contenu autre que symbolique ou l'éclatement pur et simple. Toutefois, la position particulière de Bruxelles en tant que poumon économique du pays drainant chaque matin vers elle près de 200000 personnes venues de Flandre et 100000 de Wallonie rend la deuxième option très difficile à réaliser en pratique.

\section{BIBLIOGRAPHIE}

Billiet J., 1998. Qui vote pour le Vlaams Blok? In Delwit P., De Waele J.-M., Rea A., L'extrême droite en France et en Belgique, Bruxelles, Éditions complexe.

Delwit P., 2005. Les partis régionalistes, des acteurs politico-électoraux en essor ? Performances électorales et participations gouvernementales. In Delwit P. (éd.), Les partis régionalistes en Europe. Des acteurs en développement ?, Bruxelles, Éditions de l'ULB, p 51-84.

De Decker P., Kesteloot C., De Maesschalck F. \& Vranken, J., 2005. Revitalizing the city in an antiurban context: extreme right and the rise of urban policies in Flanders, Belgium. International journal of urban and regional research, vol. $29, \mathrm{n}^{\circ} 1$.

De Winter L. \& Ackaert J., 1994. Le non-vote en Wallonie. In Frognier A.P., Aish Van Vaerenbergh A.M., Élections : la fêlure ? Enquête sur le comportement des Wallons et des francophones, Bruxelles, De Boeck Université.

De Winter L. \& Türsan H., 1998. Les partis ethno-régionalistes en Europe. Revue internationale de politique comparée, $5 / 1$.

Gijsels H. \& Vander Velpen J., 1992. Le chagrin des Flamands, Le Vlaams Blok de 1938 à nos jours, Bruxelles, Éditions EPO.

Gilissen J., 1951. Étude statistique sur la répression de l'incivisme. Revue de droit pénal et de criminologie, t. $31, \mathrm{n}^{\circ}$ 5, p. 513-628. 
Govaert S., 1993. La Volskunie. CRISP, n 1416-1417, 85p.

Gubin E., 1982. Flamingantisme et patriotisme en Belgique au XIXe siècle. Tijdschrift voor geschiedenis, 95, p. 558-576.

Hasquin H. (éd.), (1976). La Wallonie : le pays et les hommes : histoire, économies, sociétés. Tome 2. De 1830 à nos jours. Bruxelles, La Renaissance du Livre.

Klandermans B. \& Mayer N. (éd.), 2005. Extreme Right Activists in Europe. Through the magnifying glass. London, Routledge.

Lambert A., 2010. Les habitants de la région bruxelloise selon la nationalité et l'affiliation aux Communautés flamande et française : état des lieux et évolution probable. Midis de la recherche en Population et Développement, Louvain la Neuve, 23 novembre 2010.

Leton A., Miroir A., 1999. Les conflits communautaires en Belgique. Paris, PUF, 367 p.

Lothe J., 1976. Les débuts du Mouvement wallon. In Hasquin H. (éd.), La Wallonie : le pays et les hommes : histoire, économies, sociétés. Tome 2. De 1830 à nos jours, Bruxelles, La Renaissance du Livre, p 191-210.

Mabille X., 2000 (4e éd.). Histoire politique de la Belgique. Facteurs et acteurs de changement. Bruxelles, CRISP.

Marissal P., Medina Lockhart P., Van Hamme G. \& Vandermotten C., 2007. Troisième Atlas national de la Belgique. vol. 1, Géographie politique. Gand, Academia Press, SSTC et Commission de l'atlas du Comité national de géographie.

Miroir A., 1950. La Belgique et ses clivages : contradictions structurelles et familles politiques. Pouvoirs, Paris, PUF, 54, p. 5-15

Pilet J.B., 2005. Le Rassemblement wallon (RW) et le Front démocratique des francophones (FDF) : des partis victimes de leurs succès? In Delwit P. (éd.), Les partis régionalistes en Europe. Des acteurs en développement ?, Bruxelles, Éditions de l'ULB, p 265-282.

Seiler D.L., 2003. Les partis politiques. Paris, Armand Colin, 2e éd.

Van Hamme G., 2008. La géographie de l'extrême-droite peut-elle s'expliquer par l' « ethnocentrisme » des territoires ? Une analyse multiscalaire en Europe occidentale. Espace, Populations, Sociétés, 2008-3, p. 441-452.

Van Hamme G., 2009. Classes sociales et Géographie des comportements politiques en Europe occidental. Thèse de doctorat en Sciences, Bruxelles, ULB, http://theses.ulb.ac.be/ETD-db/ collection/available/ULBetd-08312009-110739

Van Haute E., 2005. La Volskunie (VU) : triomphe des idées, défaite du parti. In Delwit P. (éd.), Les partis régionalistes en Europe. Des acteurs en développement?, Bruxelles, Éditions de l'ULB, p. 243-264.

Vandermotten C., 2007. La crise de l'État belge. EchoGéo, Sur le vif, http://echogeo.revues.org/ 2049

Vandermotten C., Decroly J.M., Dessouroux C. \& Rouyet Y., 2001. Permanences et ruptures dans la géographie électorale de la Belgique. Belgeo, 2, 1-2, p. 7-40.

Vandermotten C. \& Vandeburie J., 2005. Territorialités et politique. Bruxelles, Éditions de l'ULB; Witte E. \& Craeybeckx J., 1987. La Belgique politique de 1830 à nos jours : les tensions d'une démocratie bourgeoise. Bruxelles, Labor. 


\section{NOTES}

1. Le débat est intense en Belgique sur la proportion de francophones parmi la population de la Région de Bruxelles-Capitale. Si certains hommes politiques, notamment ceux du Front des Francophones avancent un chiffre de $95 \%$ de francophones, d'autres, flamands, tablent sur une proportion de $25 \%$ de Flamands. Depuis 1961, plus aucun recensement linguistique n'a eu lieu en Belgique, ce qui est propre à polémiquer sur la question, particulièrement à Bruxelles, région bilingue. Ajoutons à cela que l'importante immigration, représentant au moins $1 / 4$ de la population, complique l'opposition francophones/néerlandophones. Les Francophones considèrent ainsi que le français est la langue prioritairement parlée par les allochtones alors que d'autres (voir l'étude de Lambert) divisent sur la question linguistique les Bruxellois en trois : francophones, néerlandophones et allochtones.

2. Afin de ne pas alimenter une controverse sur le mot flamingant qui a souvent une connotation péjorative pour les francophones, nous utiliserons le terme 'mouvement flamand' dans la suite de cet article pour parler des revendications flamandes. Le terme 'flamingant' est à l'origine utilisé pour caractériser le mouvement qui porte les revendications linguistiques et culturelles de la Flandre. Une fois obtenue l'égalité culturelle, le mouvement flamand portera d'autres revendications liées à l'autonomie flamande mais le terme en lui-même tombe progressivement en désuétude.

3. Le front de la guerre 1914-18 s'est longtemps stabilisé sur l'Yser, les troupes belges étant retirées sur cette petite portion du territoire entre l'Yser, la mer du Nord et la frontière française.

4. Précisons bien que les régions et communautés ne se confondent pas. Les premières ont une base territoriale (Flandre, Wallonie, Bruxelles) alors que les secondes ont une base culturelle : la communauté flamande, francophone (à cheval sur Bruxelles et respectivement la Flandre et la Wallonie) et germanophone, située à l'est de la région wallonne (Voir Vandermotten, 2007 pour plus de détails).

5. 1972 pour les partis libéraux et catholiques, 1978 pour le parti socialiste.

6. Spirit a, lui, formé un cartel avec le SPA (socialistes flamands).

7. Parti créé par le populiste Jean-Marie Dedecker qui obtient pour sa première participation aux élections fédérales 2007 plus de $6 \%$ des votes valables en Flandre.

8. Cette chute a été provoquée par les libéraux flamands du VLD qui avaient posé un ultimatum relatif à des enjeux communautaires. Toutefois, le VLD n'a pas pu capitaliser cette surenchère communautaire à son profit, bien au contraire.

9. Notons quand même la présence d'une liste pluri-linguistique appelée ProBXL qui souhaite promouvoir l'identité bruxelloise.

10. Chiffres issues de l'enquête Partirep réalisée conjointement par la KUL, l'UA, la VUB, l'Université de Leiden et l'ULB à l'occasion des élections régionales 2009 en Flandre et en Wallonie. Les données et analyses de ce projet sont disponibles à l'adresse suivante: http:// www.partirep.eu/

11. Néanmoins, tant pour le NV-A que pour le Vlaams Blok, cette position est déjà un compromis par rapport à leur volonté d'indépendance pure et simple.

12. Une enquête récente publiée dans la Libre Belgique du 20 octobre 2010 a globalement confirmé ces tendances avec cependant une montée apparente du sentiment d'attachement à la Flandre de la part des flamands ( $45 \%$ ).

13. Notons que ce sentiment d'appartenance à la Flandre de la part des Flamands a paradoxalement diminué depuis les années 1970. De Winter \& Frognier (2001) notent qu'à la fin des années 1970, 39 \% des Flamands se sentaient avant tout appartenir à la Flandre contre $26 \%$ en 1999, chiffre qui semble rester stable jusqu'à aujourd'hui. 
14. A titre d'illustration, la ville d'Anvers est historiquement sous-représentée dans le personnel politique de gouvernement depuis la naissance de l'État. Anvers a aussi presque toujours constitué le cœur du mouvement flamingant (Marissal \& al, 2007).

15. Notons que la radicalité du NV-A n'est pas seulement liée au régionalisme (à l'indépendantisme), qui constitue l'enjeu principal pour ce parti, mais se retrouve aussi dans les politiques d'immigration et une conception ultralibérale du point de vue socio-économique.

16. On rappelle que le vote est obligatoire en Belgique bien que les sanctions prévues soient rarement effectives.

17. La notion de "votes marginaux » recouvre selon nous la proportion d'électeurs inscrits qui ne sont pas représentés au Parlement Fédéral. Cela inclut donc les abstentionnistes, les personnes votant blanc ou ayant vu leur vote annulé et les votes pour des petits partis non représentés au Parlement.

18. Une cinquième réforme de l'Etat a lieu en 2002 mais son impact est très limité.

19. En 2003, la Cour constitutionnelle a considéré comme non-conforme à la Constitution l'existence d'un tel arrondissement bilingue qui permet aux francophones installés dans la banlieue flamande de Bruxelles de voter pour les partis francophones se présentant à Bruxelles. Depuis, les partis politiques des deux Communautés s'affrontent sur ce sujet: les Flamands s'appuient sur les arguments juridiques alors que les francophones arguent de la défense du droit des minorités.

\section{RÉSUMÉS}

Les tensions entre francophones et néerlandophones tournant autour d'une régionalisation de compétences fédérales ne sont pas nouvelles et s'inscrivent dans un lent processus de poussée régionaliste émanant aussi bien $d u$ sud que du nord du pays depuis près d'un siècle. Nous reviendrons dans cet article sur l'histoire politique et électorale du régionalisme en Belgique. Nous montrerons comment des poussées régionalistes régulières ont transformé l'État belge unitaire en un État décentralisé dans lequel le fédéral a perdu peu à peu ses prérogatives. L'hypothèse principale qui sous-tend cet article est que les partis régionalistes ont connu des succès électoraux temporaires (1919-21, 1936, 1965-77, 2009-?), suivis de déclins dès que les revendications régionalistes des opinions étaient satisfaites. La vague suivante repose alors sur un approfondissement des revendications régionalistes. Dans ce cadre, nous insisterons en particulier sur le soutien croissant des partis régionalistes au nord du pays contrastant avec l'absence de parti régionaliste wallon depuis le milieu des années 1980. Nous montrons enfin que les vagues régionalistes successives reposent sur des géographies à chaque fois renouvelées, à l'exception notable du rôle d'Anvers au sein du mouvement flamand.

The tensions between French-speaking and Dutch-speaking communities in Belgium about the regionalization of federal competences are not new and have to be understood in the long history of regionalism. This article tells the history and the geography of regional waves in Belgium. We will in particular show how these successive regionalist fevers have progressively reshaped the Belgian Central State into a federal State where regions gain in competences over the time. The main hypothesis is that regionalist parties have gone through temporary electoral successes (1919-21, 1936, 1965-77, 2009-?), followed by long period of decline as soon as the regionalist claims of the opinion were satisfied. The next wave of regionalism relies thus on the new claims 
to deepen the regionalisation of the Belgian State. In this framework, we will insist on the growing support for regionalist parties in the Flemish part of the country since the eighties, in contrast with the quasi absence of Walloon regionalist parties in the recent years. We also show that the geography of regionalist parties have considerably evolved over the time, with the exception of Antwerp which has remained at the heart of most Flemish regionalist parties.

\section{INDEX}

Keywords : Belgium, electoral geography, Flemish movement, regionalism

Mots-clés : Belgique, géographie électorale, mouvement flamand, régionalisme

\section{AUTEURS}

\section{GEOFFREY PION}

Geoffrey Pion, geofpion@ulb.ac.be, Chercheur à l'IGEAT-ULB, a publié récemment :

- Pion G., 2009. Géographie des comportements de vote protestataires en Wallonie : une approche infra-communale à Charleroi et Mons. Belgeo, 2009, $n^{\circ}$ 2, p 153-168

- Pion G., 2010. Le vote blanc et nul en Wallonie : analyse écologique et individuelle. Belgeo, 2010, $n^{\circ} 3$, à paraître.

\section{GILLES VAN HAMME}

Gilles Van Hamme, gvanhamme@ulb.ac.be, Chercheur-assistant à l'IGEAT-ULB, a publié récemment :

- Van Hamme G., 2008. La géographie de l'extrême-droite peut-elle s'expliquer par l' « ethnocentrisme » des territoires ? Une analyse multiscalaire en Europe occidentale. Espace, Populations, Sociétés, 2008-3, p. 441-452.

- Van Hamme G., 2010. Le dilemme géographique des partis sociaux-démocrates : l'exemple des partis socialistes en Belgique. Cybergeo, Espace, Société, Territoire, article 487, mis en ligne le 03 février 2010, URL : http://www.cybergeo.eu/index22902.html 